\title{
REDES CLARA E SCIENTI: TECNOLOGIA E INFORMAÇÃO PARA A CONSTRUÇÃO DO CONHECIMENTO NA COMUNIDADE CIENTÍFICA IBÉRO-AMERICANA
}

\section{CLARA AND SCIENTI NETWORKS: TECHNOLOGY AND INFORMATION FOR KNOWLEDGE BUILDING IN THE LATIN AMERICAN SCIENTIFIC COMMUNITY}

\author{
Rejane Sartori ${ }^{1}$; Roberto Carlos dos Santos Pacheco ${ }^{2}$ \\ ${ }^{1}$ Universidade Federal de Santa Catarina - UFSC - Florianópolis; Universidade Estadual de Maringá \\ - UEM; Centro Universitário de Maringá - Cesumar - Maringá; Fafijan - Jandaia do Sul - Brasil \\ rejane@egc.ufsc.br \\ ${ }^{2}$ Universidade Federal de Santa Catarina - UFSC/INE - Florianópolis - Brasil pacheco@ stela.org.br
}

\begin{abstract}
Resumo
O desenvolvimento baseado no conhecimento gera riqueza abrindo fronteiras para a competitividade, inovação tecnológica e distribuição de renda. Em países desenvolvidos esse processo está intrinsecamente ligado à capacidade de gerar inovação e à dinâmica de construção de conhecimento em redes. Nesse processo, as comunidades acadêmicas e de pesquisa participam efetivamente na dinâmica de geração de conhecimento e inovação, um ambiente de trabalho fortemente baseado em TICs. Na América Latina, entretanto, a formação de tais comunidades é reduzida, quando comparada à dinâmica de países desenvolvidos. É nesse contexto que a Rede CLARA (Cooperação Latino Americana em Redes Avançadas) e a Rede ScienTI (Rede de Informação e Conhecimento em Ciência, Tecnologia e Inovação) surgem como mecanismos de criação, disseminação e promoção de conhecimento científico na região ibero-americana. A Rede CLARA integra as redes acadêmicas nacionais da América Latina, conectando mais de 700 universidades e centros de pesquisa nessa região. A Rede ScienTI forma e conecta fontes de informação científica de 11 países da região. Esse cenário põe em perspectiva a formação de uma gigantesca comunidade de pesquisa latino-americana, conectando pesquisadores, projetos, estudos e pesquisas, cumprindo assim importante papel na construção do futuro em ciência e educação. Nesse artigo, discutem-se as criações e funcionamentos dessas redes sob a perspectiva de construção de conhecimento em comunidade na região ibero-americana.
\end{abstract}

Palavras-chave: tecnologia e informação, comunidade científica, redes acadêmicas e de pesquisa, américa latina, conhecimento.

\section{Introdução}

As Tecnologias da Informação e das Comunicações (TICs) se tornaram elemento intrínseco à construção da Sociedade do Conhecimento. Compreender como essas tecnologias estão 
revolucionando a Ciência, a Economia e a Sociedade como um todo se constitui em agenda de estudos para importantes centros de pesquisa e diversas organizações internacionais.

Estudiosos da conjuntura atual afirmam que a sociedade transformou-se em uma sociedade em rede. Segundo Castells (1999), as redes constituem a nova morfologia social de nossas sociedades, e a difusão da lógica de redes modifica de forma substancial a operação e os resultados dos processos produtivos e de experiência, poder e cultura. Para esse autor, o novo paradigma da tecnologia da informação fornece a base material para a inserção crescente das redes em toda a estrutura social.

Assim, na ciência, a imagem do cientista como um ser isolado e absorto em suas pesquisas faz parte do passado. $\mathrm{Na}$ atualidade, o processo de produção do conhecimento científico requer competência, associação, cooperação, negociação, diretrizes e estratégias que visem interligar o maior número de elementos de forma a promover a construção do conhecimento (SILVA, 2005). Nesse contexto, há uma dependência de recursos de infra-estrutura. Busca-se um sistema de pesquisa e desenvolvimento que, ao mesmo tempo, viabilize o avanço do conhecimento científico e tecnológico e propicie a formação, em grande escala, de recursos humanos altamente qualificados, importantes para o próprio sistema de pesquisa e para os quadros das empresas (TIDIA, 2005).

Segundo Melo e Machado (2004), é essencial assegurar uma efetiva participação das comunidades nacionais de pesquisa na dinâmica dos processos de geração de conhecimento e inovação. De acordo com esses autores, os países da América Latina apresentam um quadro ainda muito heterogêneo e incipiente com relação à infra-estrutura de TICs para pesquisa, em particular em termos de redes digitais avançadas. Isso se evidencia quando da comparação com outras regiões mais desenvolvidas do mundo. As TICs podem tornar possível acompanhar as tendências e identificar oportunidades de usos e de desenvolvimento de aplicações especiais orientadas às diversas áreas do conhecimento.

Neste contexto, há dois cenários novos e promissores para a região ibero-americana: a consolidação da Rede de Cooperação Latino Americana em Redes Avançadas (Rede CLARA) e a formação da Rede de Fontes de Informação e Conhecimento em Ciência, Tecnologia e Inovação (Rede ScienTI). A primeira estabelece a infra-estrutura de conexão das redes acadêmicas da região, enquanto a segunda está permitindo a formação de fontes nacionais de informação sobre Ciência, Tecnologia e Informação (CT\&I) homogêneas e interoperáveis em toda a região.

Neste artigo discute-se a importância das redes de cooperação científica e das redes acadêmicas na Sociedade do Conhecimento, as redes existentes na América Latina e o novo cenário que as Redes CLARA e ScienTI trazem à região como mecanismos de promoção e desenvolvimento do conhecimento na sua comunidade científica. 


\section{A Sociedade do Conhecimento}

O início do século XXI está sendo marcado por mudanças nos níveis sociais, econômicos, políticos, culturais e institucionais. Tem sido consenso o fato de o conhecimento não ser mais um meio adicional de riquezas, mas sim o fator dominante. A formação de grandes blocos econômicos e a integração de diferentes mercados financeiros, decorrentes da globalização, são apenas a face econômica dessa nova sociedade, que tem como "pano de fundo" o crescimento acelerado dos setores mais intensivos de informação, fundamentados na conectividade e cada vez mais na interoperabilidade tecnológica. A riqueza concentra-se, então, na capacidade e na velocidade com que informações são transformadas em ações, ou seja, em conhecimento.

O conceito da transformação de informações em ações foi previsto por Drucker (1992) quando afirmou que as tecnologias, por si mesmas, não gerariam uma maior produtividade no trabalho. Para esse autor, na nova economia, a atual regra é estar preparado para competir com competência, mesmo porque o passado não mais irá se repetir. O sucesso de ontem já não garante mais o sucesso de hoje e, conseqüentemente, não sustentará o sucesso de amanhã.

Assim, nesta nova sociedade, reconhece-se como riqueza o conhecimento, que se tornou a fonte de mais alto impacto ao desenvolvimento, e a busca pelos mecanismos de riqueza projeta-se no controle dos meios de comunicação e disseminação de informação. As TICs são fatores de riqueza somente quando a infra-estrutura de comunicação alia-se à competência de transformação da informação em conhecimento. É nesse contexto que a educação é o elemento-chave para a construção da Sociedade do Conhecimento, na qual inovar e converter conhecimento em vantagem competitiva são os mais importantes diferenciais (FREITAS JUNIOR, 2003).

Além do fator de transformação dos meios de produção, outra questão-chave na Sociedade do Conhecimento é o fator de escala. Às TICs está posto o desafio de conectar cada vez mais pessoas em projetos de cooperação que promovam conhecimento. É nesse contexto que estão as redes de cooperação científica. Aliadas as TICs, a clássica forma de se produzir conhecimento em comunidade ganha fator de escala, conecta realidades complementares e promove desenvolvimento.

\section{Redes de Cooperação e Fontes de Informação Científica}

A colaboração científica é um fenômeno tão antigo quanto a própria ciência. A cooperação envolvendo pesquisadores de países diferentes foi detectada já no século XIX.

De forma genérica, a colaboração científica é um empreendimento cooperativo que envolve metas comuns, esforço coordenado e resultados ou produtos através dos quais os colaboradores compartilham a responsabilidade e o crédito (BALANCIERI, 2004). Podendo ser encontrada nas 
mais diferentes formas, a colaboração científica se dá, freqüentemente, no âmbito dos chamados “colégios invisíveis", identificados nos anos 60 por Price (1963) e que se constituíram em comunidades informais de cientistas que, comunicando-se e reunindo-se periodicamente para a troca de experiências e informações, passaram a desempenhar papel fundamental na publicação formal e na disseminação dos avanços no campo do conhecimento científico.

Na visão de Velho (2001), estes cientistas são caracterizados por sua alta produtividade, por compartilhar prioridades de pesquisa, por treinar estudantes, por produzir e monitorar o conhecimento em seu campo. Encontram-se em congressos, conferências, reuniões sobre suas especialidades, visitam-se por meio de intercâmbios institucionais ou realizam trabalhos em colaboração. Este tipo de organização transcende os limites do departamento, da instituição, de um país, e abrange cientistas de todos os lugares do mundo onde tiver atividade científica relevante na área, ou na especialidade em questão.

As redes informais que os cientistas estabelecem entre si são partes essenciais do conhecimento científico. Para Macedo (1999), o produto final, freqüentemente expresso por meio da publicação dos resultados produzidos pelos pesquisadores envolvidos, é resultado da série de informações e conhecimentos que são trocados informalmente e consolidados ao longo do tempo pelas várias comunidades científicas, permitindo ainda identificá-los, bem como identificar suas instituições e seus países de origem.

Segundo Velho (2001), nas últimas décadas a intensificação das atividades da Big Science, através de seu impacto na organização social de algumas áreas do conhecimento, provocou o estabelecimento e funcionamento de grandes laboratórios e grandes projetos biológicos, exigindo a participação de grupos de pesquisa localizados em países diferentes - não apenas pela complexidade científica, mas principalmente pelos custos envolvidos - provocando significativa elevação dos índices de cooperação internacional.

Mais recentemente, ainda segundo esse autor, dois acontecimentos têm contribuído para modificar este padrão de cooperação científica internacional. De um lado, as preocupações com problemas que extrapolam as fronteiras geográficas (e.g., efeito estufa, rompimento da camada de ozônio), de outro, o crescimento de setores industriais de alta tecnologia, que estimula a parceria científica entre empresas e entre países desenvolvidos. Tais arranjos permitem às grandes empresas transnacionais, a partir de investimentos conjuntos em pesquisa científica, gerar, também conjuntamente, novas oportunidades tecnológicas e, em separado, capacitar-se para explorá-las. Incentivada pelos governos através da criação de programas especiais, esta nova forma de cooperação está se tornando típica entre os países avançados.

Portanto, dois fatores são notadamente favoráveis à formação de redes em ciência: o primeiro está na própria forma com que o conhecimento científico se articula, intrinsecamente 
ligado à cooperação entre grupos de pesquisa e pesquisadores; o segundo fator está na ampliação das possibilidades de realizar cooperação, com o surgimento dos novos recursos em TICs. A combinação de ambos tem feito surgir as redes acadêmicas de pesquisa.

\subsection{Redes Acadêmicas e de Pesquisa}

As Redes Acadêmicas e de Pesquisa têm seu contexto nas transformações econômicas, sociais, políticas e tecnológicas das últimas décadas. A introdução do computador, a adoção de técnicas e ferramentas de modelagem e de simulação e, sobretudo, o surgimento da Internet, representaram fatores de transformação do processo de geração do conhecimento, incluindo-se a organização da atividade de pesquisa (MELO; MACHADO, 2004).

Segundo Weisz e Roco (1996), essas transformações levaram ao encurtamento do ciclo pesquisa-desenvolvimento-produto. As demandas por pesquisas incluem a formação de base científica ampla, globalização crescente da ciência e abertura dos mercados à competição global. Novas idéias científicas, a criação de novas tecnologias e a formação de bons profissionais suplantaram a possibilidade de existência de instituições isoladas. Ademais, as crescentes restrições orçamentárias, a existência de centros distintos com capacidades semelhantes, a submissão internacional da ciência e tecnologia e a disponibilidade de melhores meios de comunicação resultaram na formação de redes de atividades de pesquisa.

Uma rede de pesquisa é formada por pesquisadores que trocam entre si informação e conhecimento e que têm como produto final algum tipo de produção: bibliográfica, tecnológica, artística ou cultural. Os principais objetivos dessas redes são a pesquisa, a formação, o desenvolvimento tecnológico cooperativo, a cooperação externa e as atividades interdisciplinares (BALANCIERI, 2004).

A efetivação das redes de pesquisa se deu a partir das telecomunicações, com a inserção de mecanismos que permitiram aos pesquisadores colaborarem e partilharem informações e recursos. Segundo Stanton (2005a), esses mecanismos incluem redes de comunicação de alto desempenho com recursos computacionais distribuídos, normalmente providos por um grande número de computadores individuais, ou de um conjunto destes, que juntos colaboram na solução de problemas maiores - as chamadas grades computacionais.

As características dessas redes, de acordo com Melo e Machado (2004), constituem um indicativo da capacidade de transferência e compartilhamento de dados entre países. Por outro lado, ao permitirem elevadas taxas de transmissão de dados e interoperação entre computadores situados em espaços geográficos diversos, estabelecem as bases para o desenvolvimento de um ambiente de 
colaboração entre grupos de pesquisa que atuam em localidades distintas, mesmo as geograficamente mais remotas.

Neste contexto, as redes de pesquisa têm uma finalidade multifuncional com dois objetivos principais: (a) funcionam como uma infra-estrutura de alta capacidade de informação e comunicação com base nas tecnologias mais avançadas para apoiar o trabalho dos pesquisadores; e (b) facilitam a pesquisa, proporcionando uma plataforma para implementar novos serviços e tecnologias avançadas de rede. Sempre que houver desenvolvimentos tecnológicos de vanguarda, as redes de pesquisa estarão numa posição ideal para testar novos serviços antecipadamente ao mercado de telecomunicações (CLARA, 2005).

Melo e Machado (2004) lembram que esse novo ambiente digital é caracterizado por crescente capacidade de armazenamento e de processamento de informação e interatividade. Com isso, vem permitindo aplicações revolucionárias (e.g., acesso de informações em tempo real e instrumentação remota), acesso a grandes infra-estruturas existentes em apenas alguns países (e.g., observatórios astronômicos ou aceleradores de partículas), compartilhamento de dados e processamento distribuído de informações. Permitirão avanços consideráveis nos diversos campos do conhecimento e também na pesquisa de interesse industrial.

Novas tecnologias de comunicação sem fio, transmissões óticas e novos paradigmas tecnológicos, como o advento das nanotecnologias, transformarão ainda mais o cenário da pesquisa no mundo nos próximos anos. Melo e Machado (2004) ressaltam os vultosos investimentos anunciados pelos países desenvolvidos nessa nova infra-estrutura para pesquisa, a exemplo do Advanced Cyberinfrastructure Program da National Science Foundation (NSF), nos Estados Unidos.

$\mathrm{Na}$ União Européia isso pode ser constado em seu maior programa de desenvolvimento, o Programa IST (Tecnologias da Sociedade de Informação), que há vários anos fomenta um largo espectro de atividades de pesquisa e desenvolvimento em TICs (STANTON, 2005a). Há, portanto, preocupação especial quanto à infra-estrutura eletrônica. Seu foco está na melhoria da rede GÉANT, a rede pan-européia de alta velocidade, e em montar uma capacidade computacional distribuída baseada em grade, capaz de prestar serviços para a ciência eletrônica em regime de produção.

Considerada a rede mais avançada do mundo no seu gênero e a que oferece maior cobertura (interliga mais de 3.500 instituições de ensino e pesquisa em 32 países), a GÉANT é apenas a parte mais visível do investimento recente dos europeus em comunicação de alto desempenho (STANTON, 2005a). Além de investimentos em sua própria infra-estrutura, a União Européia vem investindo em promover novas conexões para outras regiões do mundo: África, Oriente Médio, Ásia Central, Ásia Oriental e América Latina. Visa-se, com isso, colocar a Europa numa posição de destaque no mundo da comunicação digital. 


\subsection{Fontes de Informação em Ciência, Tecnologia e Inovação}

Além de conectar laboratórios e grupos de pesquisa, a promoção do trabalho cooperativo em rede requer acesso a fontes de informação. Essas devem, além de permitir conhecer fontes de financiamento, ser fonte para o autoconhecimento da própria comunidade científica. As perguntaschave na composição de redes de pesquisa são: (a) Quem - que grupos e especialistas estão trabalhando com determinado conhecimento?; (b) Onde - em que local e instituição estão esses especialistas?; (c) $O$ que - qual é a contribuição que esses grupos trazem à área do conhecimento em que atuam?; (d) Como - de que forma essas contribuições vêm sendo alcançadas (projetos, produção científica)? e (d) Com quem - que outros grupos e especialistas cooperam nas atividades?

Visando responder a essas questões, diversos investimentos têm sido realizados por organizações nacionais e internacionais ligadas a CT\&I. Na Europa destacam-se o projeto CORDIS (Community Research \& Development Information Service) ${ }^{1}$, o EuroCris (Current Research Information Systems) ${ }^{2}$ e o CERIF (Common European Research Information Format). CORDIS é uma iniciativa da União Européia que organiza e disponibiliza informações sobre CT\&I, incluindo serviços de procura por parceiros, projetos, fontes de financiamento, produção científica (monografias, documentos e artigos científicos). EuroCris é uma associação européia sem fins lucrativos que objetiva atuar como fórum para todos indivíduos e organizações interessados no uso de tecnologia de informação na condução de toda informação de pesquisa nos sistemas de negócio. CERIF é resultado do trabalho de um grupo de especialistas dos estados membros da União Européia que estabelece padrões e formatos comuns para informações relacionadas à pesquisa, trabalho que assume importância no contexto da interoperabilidade de fontes de informação.

\subsection{Redes Acadêmicas e de Pesquisa e Fontes de Informação na América Latina}

O momento é de avanço expressivo na capacidade e na conexão internacional das redes acadêmicas e de pesquisa nas diversas regiões do mundo, incluindo-se redes nacionais, regionais e locais, nos mais diversos países. Melo e Machado (2004) consideram que essas redes cada vez mais se constituem em elemento focal para as estratégias de promoção do desenvolvimento econômico e social, a exemplo do que ocorre em países da Europa Oriental e em países asiáticos.

$\mathrm{Na}$ América Latina, uma extensa área geográfica que abriga mais de 700 universidades e institutos de pesquisa, o cenário é ainda incipiente e se observa uma heterogeneidade em relação à presença das redes acadêmicas e de pesquisa. Segundo Melo e Machado (2004), fatores como o

\footnotetext{
1 www.cordis.lu

2 www.eurocris.org
} 
elevado custo dos serviços de comunicação e a retração de investimentos nos sistemas nacionais de CT\&I, particularmente nas universidades e nos centros públicos de pesquisa, contribuem para este cenário de desvantagem em relação a outras regiões.

A iniciativa de interligar as redes nacionais de países da América Latina para estabelecer a conexão com a GÉANT, chamada ALICE (America Latina Interconectada Con Europa), foi a responsável pela montagem da rede CLARA (Cooperación Latino Americana de Redes Avanzadas), que se constitui em importante integração entre as redes nacionais acadêmicas e de pesquisa da região.

No âmbito das fontes de informação, na América Latina destacam-se duas iniciativas: (a) Rede RICYT - Red Iberoamericana de Indicadores de Ciencia y Tecnologia ${ }^{3}$; e (b) INFOCYT (Red de Información C\&T para América Latina y el Caribe). RICYT estabelece padrões e produz indicadores em CT\&I para a região, de forma semelhante ao trabalho da Organização para Cooperação e Desenvolvimento Econômico (OCDE). INFOCYT é uma iniciativa da Organização dos Estados Americanos (OEA) que estabeleceu um Portal com links para as fontes de informações sobre C\&T na América Latina e Caribe.

Os cenários internacionais, portanto, tanto em países desenvolvidos como na região latinoamericana apontam para o aprofundamento das redes de pesquisa em duas frentes: (a) conectividade da infra-estrutura em TICs disponível nos países; e (b) formação e interoperabilidade entre fontes de informação em CT\&I. Nesse sentido, os principais projetos na região nessas duas frentes são, respectivamente, a Rede CLARA e a Rede ScienTI, descritas a seguir.

\section{Rede CLARA}

A Rede CLARA tem gênese em evento do programa Alliance for the Information Society, patrocinado pela União Européia, realizado em junho de 2002. Naquela ocasião, os representantes das principais redes acadêmicas latino-americanas se encontraram diante da oportunidade de constituir a rede latino-americana que tantas vezes tinha-se tentado fundar (RNP, 2005). Um ano depois, representantes das redes acadêmicas de 18 países da América Latina assinaram a ata de constituição da organização e em agosto de 2004 a Rede CLARA começou a operar, sendo hoje mecanismo de facilitação de trabalho das redes acadêmicas avançadas da região (RNP, 2005).

Com sede no Uruguai, CLARA é uma associação civil, sem fins lucrativos que tem duas frentes de atuação: (a) a formação de uma infra-estrutura que una as redes avançadas latinoamericanas; e (b) a gestão da organização não governamental que represente os interesses da rede. Esta Rede foi planejada e está sendo implementada a partir das redes nacionais de educação e

\footnotetext{
3 www.ricyt.org
} 
pesquisa dos países que a compõem, e conta ainda com conexão com as redes comerciais, permitindo o acesso eficiente por qualquer usuário a partir de acordos de interconexão entre as redes. Apenas uma rede por país pode ser membro da CLARA, a qual deve ser explicitamente apoiada pelo governo de seu país. Seus objetivos estratégicos incluem a coordenação com outros blocos, a cooperação para a promoção do desenvolvimento científico e tecnológico, o planejamento e a implantação de serviços de redes para a interconexão regional, culminando no desenvolvimento de uma rede regional (Rede CLARA) para interconectar as redes nacionais acadêmicas e de pesquisa que serão operadas por seus associados (CLARA, 2005).

Atualmente CLARA inclui as seguintes redes: RETINA (Argentina), ADSIB (Bolívia), RNP (Brasil), REUNA (Chile), Universidad de Cauca (Colombia), CR2Net (Costa Rica), RedUniv (Cuba), RAICES (El Salvador), CEDIA (Equador), RAGIE (Guatemala), UNITEC (Honduras), CUDI (México), CNU/RENIE (Nicarágua), RedCyT (Panamá), ARANDU (Paraguai), RAAP (Peru), RAU (Uruguai) e REACCIUN (Venezuela) (CLARA, 2005). Com essa configuração, CLARA conecta atualmente mais de 700 universidades e centros de pesquisa em países na América Latina e Caribe.

A topologia da Rede CLARA (Figura 1) contempla as principais redes nacionais da região (Argentina, Chile, Brasil e México) e o acesso aos países da América Central. Além disso, a Rede será conectada à Rede GÉANT através de uma conexão saindo do Brasil e outra saindo do México para a Califórnia, dando acesso ao Canadá, Estados Unidos e Ásia (CLARA, 2005). Essas adesões, na visão de Melo e Machado (2004), possibilitam a formação de grupos de pesquisa interinstitucionais com universidades, centros de pesquisa e agências nacionais entre os países que configuram a Rede e permitirão o uso de aplicações comuns de pesquisa e ensino em diversas áreas do conhecimento, como Medicina, Física, Astronomia, Saúde, Meio Ambiente, Educação, Biodiversidade, entre outras. 


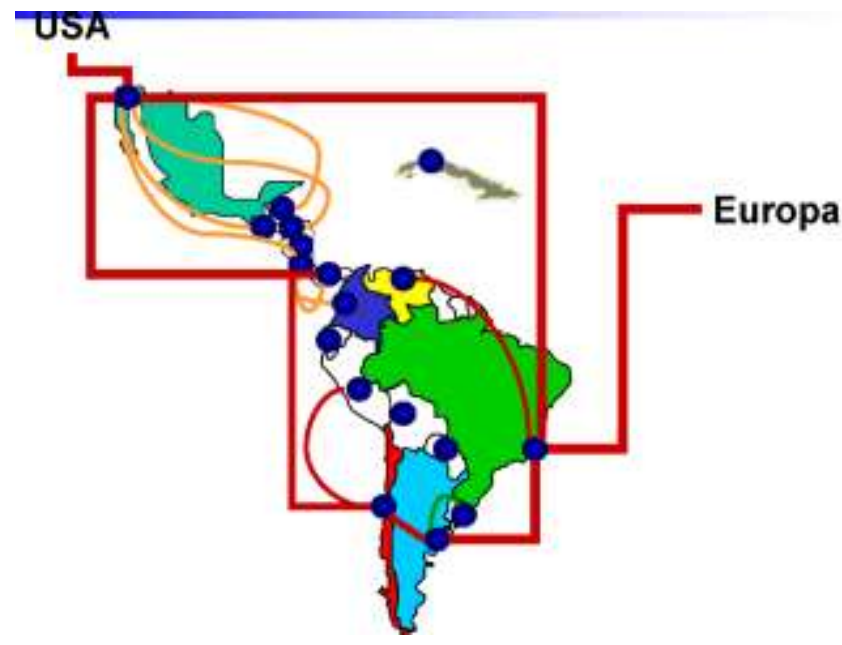

Fonte: CLARA (2005).

As atividades de CLARA atualmente são: (a) apoio às redes membros; (b) implementação e gerência da Rede; (c) formação de base de dados de grupos de pesquisadores e especialistas; e (d) criação de grupos de segurança (CLARA, 2005).

Segundo Simões (2005), é a primeira vez que os países da América do Sul estão conectados por uma rede avançada. Além disso, a conexão com a Europa permite que se abra uma agenda ampla entre essas duas partes do mundo, em termos de colaboração científica. Stanton (2005b) afirma que os benefícios a curto prazo incluem novo acesso às redes avançadas internacionais, com melhores facilidades de comunicação com as redes européias e com as dos países latinoamericanos. Espera-se que esta rede possa crescer para atender as necessidades internacionais para colaboração com pesquisadores, laboratórios e observatórios localizados nos países da região.

Pesquisadores e acadêmicos latino-americanos poderão realizar projetos colaborativos de pesquisa e educação que transcendam as fronteiras nacionais. Essa Rede representa não somente um significativo passo à frente para o desenvolvimento da infra-estrutura de redes de pesquisa através da América Latina e desta última com a Europa, mas torna realidade o posicionamento da região no mais alto nível de desenvolvimento científico e tecnológico (CLARA, 2005).

Com todas as potencialidades que a Rede CLARA traz para a comunidade acadêmica e de pesquisa latino-americana e sua projeção de cooperação com a Europa, requer ferramentas de informação que apóiem a busca dos objetivos de colaboração esperados. Neste sentido, planeja-se desenvolver ACLARA, um Catálogo Padronizado de Projetos de P\&D (Pesquisa e Desenvolvimento) latino-americano, realizando assim um serviço de informação padronizado transregional que permita às instituições pertencentes à CLARA estabelecer um repositório em um esquema distribuído por país e padronizado com informações relevantes dos projetos de pesquisa latino-americanos (com base em adoção de metadados de EuroCris e CERIF). A meta é servir como 
estrutura de informação pública e única a serviço dos interesses das instituições associadas a esta iniciativa. A coordenação da REUNA (Chile), e seus serviços de administração e desenvolvimento de sistemas de informação, apoiada pela infra-estrutura da rede CLARA, irá articular a criação dessa base de informação para apoio à realização de projetos colaborativos de pesquisa usando as TICs (CLARA, 2005).

Em termos de apoio direto à cooperação científica, Geraque (2005) menciona que a Rede CLARA possibilitará monitorar um telescópio de última geração instalado na Cordilheira dos Andes sem sair de uma sala em São Paulo; participar no Rio de Janeiro de projetos de computação compartilhada que estão sendo desenvolvidos em Paris; fazer uma videoconferência entre Brasília e Lisboa sem ter que perder preciosos milissegundos na transmissão, uma vez que os sinais digitais não precisarão mais passar, como antes, pelos Estados Unidos.

Essas iniciativas, na visão de Cabral (2005), podem favorecer a muitos na América Latina. Pela disposição no trabalho que vem sendo implementado pelas pessoas e organizações envolvidas na América Latina, o que mais se deseja é que essas redes sejam conhecidas e gerem projetos de vida longa para o benefício de toda a sociedade.

Para Melo e Machado (2004), a criação da Rede CLARA se constitui marco importante por facilitar o desenvolvimento e implantação de redes onde ainda não existem e gerar oportunidades para a construção de uma agenda de pesquisa de interesse regional e global. Até muito recentemente, os elevados custos das telecomunicações restringiam as possibilidades de conexão intra-regional na América Latina, mas hoje é possível para um conjunto de países planejar conectividade na América Latina ao mesmo valor que entre cada país e Miami, por exemplo. Além da autonomia e da capacitação gerada e difundida, a rede intra-regional permite a expansão de aplicações sensíveis, ou de controle remoto ou assistido, com tempos menores do que atualmente observados (com a infra-estrutura existente e em operação) na propagação de grandes massas de dados. Assim sendo, com essa rede intra-regional será possível assegurar alta qualidade em aplicações de colaboração à distância (educação, saúde etc.) e suporte a futuros grandes projetos intra-regionais em áreas de grande complexidade científica e tecnológica.

Com relação a sustentabilidade da Rede, para sua montagem e manutenção até 2006, a Comissão da União Européia está financiando até $80 \%$ das despesas, num montante de 12,5 milhões de euros. Em cada país é ainda necessário contar com o apoio explícito do governo nacional e às vezes negociar tratamento tarifário diferenciado de operadoras de telecomunicações, em função da finalidade não comercial dessa rede. Finalmente, cada rede nacional precisa assumir a responsabilidade para o pagamento da sua parte dos $20 \%$ restantes que cabem aos beneficiários latino-americanos do projeto (CLARA, 2005). A sustentabilidade da CLARA depende de encontrar 
formas alternativas de financiamento e todas estas combinações ainda levarão certo tempo para serem concluídas.

\section{Rede ScienTI}

A Rede $\mathrm{ScienTI}^{4}$ (Rede Internacional de Fontes de Informação e Conhecimento em Ciência, Tecnologia e Inovação) tem gênese no acordo firmado no ano de 2000 entre o Conselho Nacional de Desenvolvimento Científico e Tecnológico (CNPq) do Brasil e a Organização Pan-americana de Saúde (OPS). O acordo teve por finalidade combinar as experiências dessas organizações em dois projetos de criação e gestão de fontes de informação em CT\&I: Plataforma Lattes do CNPq e Biblioteca Virtual de Saúde (BVS) da OPS/BIREME. De um lado, a Plataforma Lattes sistematizou parte do conjunto de informações de um sistema nacional de inovação (currículos, grupos de pesquisa, instituições e projetos). De outro, a BVS se transformou no principal espaço de acesso a fontes de informação (bibliotecas digitais) e de cooperação em saúde na América Latina e Caribe.

A combinação dessas experiências se deu pela construção de uma arquitetura baseada em padrões e nos sistemas de informação da Plataforma Lattes (PACHECO; KERN, 2003). Em 2001, CNPq, OPS e Grupo Stela, da Universidade Federal de Santa Catarina, elaboraram um sistema piloto (CVLACS - Curriculum Vitae Latino-Americano em Ciências da Saúde) a partir da tradução do CV-Lattes brasileiro. Após uma aplicação em Chile, Colômbia, Cuba, México e Venezuela, gerenciada pelos Órgãos Nacionais de Ciência e Tecnologia (ONCYTs) daqueles países, o êxito da experiência levou à formalização de uma Rede, com a adesão de Portugal, Peru, Panamá, Equador e Argentina, além de UNESCO e RICYT como organismos internacionais. Em dezembro de 2002, a Rede foi formalizada em reunião realizada em Florianópolis, Santa Catarina.

Administrativamente, a Rede ScienTI está estruturada em quatro núcleos: (a) ONCYTs, representando os governos nacionais que adotam a arquitetura ScienTI e são responsáveis pelas fontes nacionais de informação em CT\&I; (b) OICTYs, incluindo as organizações internacionais de apoio à CT\&I (incluindo OPAS, UNESCO, RICYT); (c) GDIs - Grupos de P\&D em sistemas de informação aplicados à CT\&I (incluindo Stela-Brasil, CT\&S-Universidade Nacional de Colômbia, Quipu/PUC-Peru e DSI-Universidade do Minho-Portugal); e (d) IPs - Núcleo de Instituições Promotoras da Rede. Além disso, a Rede possui uma Secretaria Executiva (com mandato atual de OPAS/BIREME) e um Comitê Interinstitucional, formado por representantes de seus núcleos e responsável por estabelecer as diretrizes da Rede.

O principal objetivo da Rede ScienTI é criar um espaço internacional de CT\&I baseado em fontes de informação e conhecimento para gestão da atividade científica e inovação tecnológica.

\footnotetext{
4 www.scienti.net
} 
Trata-se de um espaço de interação e uma plataforma aberta operando em rede de sistemas de informação, diretórios, portais Web e ferramentas de extração de dados e informação. A Rede é uma expressão cooperativa internacional entre os ONCYTs, OICYTs e GDIs para o desenvolvimento contínuo de metodologias e ferramentas que apóiem a gestão da atividade científica e tecnológica dos países e, em particular, dos países em desenvolvimento.

Em cada país, a Rede ScienTI permitiu a formação de fontes nacionais de informação em CT\&I. Seus sistemas já são utilizados oficialmente no Brasil (mais de 580 mil currículos e acima de 20 mil grupos de pesquisa), Colômbia (mais de 40 mil currículos e mais de 2 mil grupos), Venezuela (cerca de 10 mil currículos), Equador (cerca de 4 mil currículos), Argentina (cerca de 3 mil currículos) e Chile (aproximadamente 4 mil currículos). Além desses, estudam procedimentos de implantação Peru, Portugal e Panamá, além de México, Paraguai e Uruguai (atualmente observadores na Rede).

As fontes de informação são geradas em cada país, sob a gestão do ONCYT, com o apoio do GDI nacional (quando existente). Elas surgem da inserção dos sistemas ScienTI no processo de fomento a C\&T, baseado no envio de currículos e grupos de pesquisa (em alguns países) para o ONCYT. Na estrutura do ONCYT web services e mecanismos de interoperabilidade, publicam as informações para consultas externas (Figura 2). No Portal ScienTI, as consultas são acessadas de forma integrada, de modo a permitir que a busca por um especialista seja propagada para toda a região.

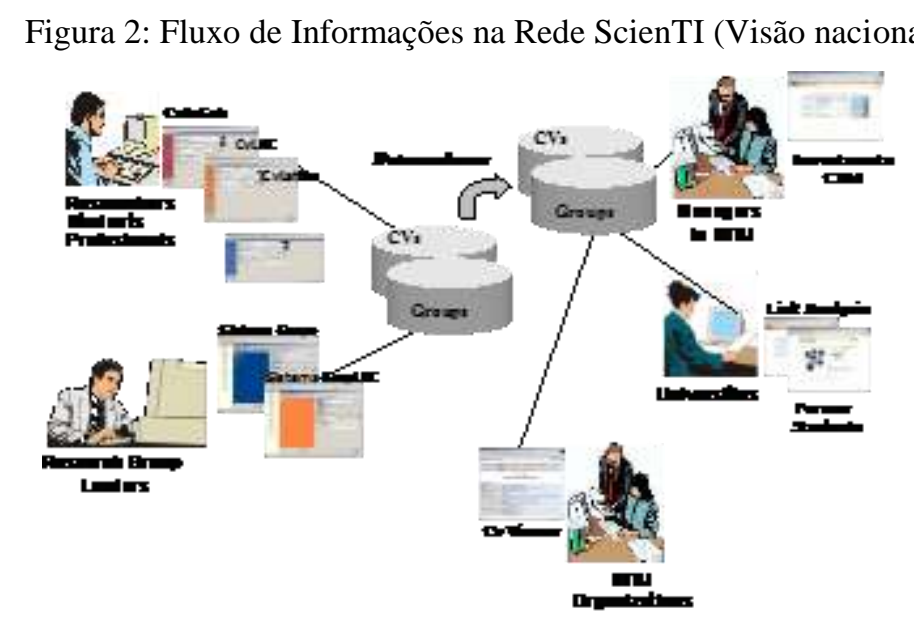

Fonte: SCIENTI (2005)

Além de disponibilizar o acesso às fontes de informação, ScienTI possui interoperabilidade com bibliotecas digitais (BVS, SciELO, LILACS, MedLine) e tem viabilizado estudos cientométricos em saúde (realizados por OPAS e RICYT). Em suas perspectivas está a formação de comunidades de prática, em temas específicos ligados à saúde. Em perspectiva, também se 
evidencia a aplicação de sistemas de conhecimento para tornar mais efetiva a busca por especialidades em CT\&I (GONÇALVES et. al., 2005).

Ao contrário da Rede CLARA, a Rede ScienTI é individualmente mantida por cada organismo participante. Entre as perspectivas de sustentabilidade está a possibilidade de se criarem cortes temáticos na Rede, a ampliação de parceiros e o encaminhamento de projetos específicos a órgãos de fomento (a exemplo do que Colômbia realizou junto à OEA).

Para a região, ScienTI pode representar o acesso imediato a informações sobre a comunidade científica. Saber quem, onde, em que e com quem se realizam as pesquisas na América Latina é a principal contribuição que a Rede ScienTI tem a oferecer à promoção do conhecimento e do desenvolvimento na região.

\section{Considerações Finais}

É inegável o papel que as TICs podem desempenhar no processo de construção e disseminação do conhecimento, estreitando assim a colaboração internacional na produção de conhecimento. O intercâmbio de experiências e a colaboração no processo de conhecimento intensifica a busca pela ciência sem fronteiras e, sobretudo, contribui para a busca do desenvolvimento sustentável em perspectiva de cooperação internacional.

Esse intercâmbio favorecido pelas novas TICs pode contribuir para elevar a capacidade de pesquisa, a capacitação para a pesquisa e o acesso ao conhecimento. Essa já é uma realidade crescente nas redes acadêmicas e de pesquisa, em que instituições e pesquisadores envolvidos em atividades de pesquisa atuam cooperativamente na geração de conhecimento e inovação, especialmente em países mais desenvolvidos. A criação de infra-estrutura eletrônica aos níveis nacional e internacional aprofundou os laços de cooperação remota entre os cientistas que dependem da abundância de recursos computacionais para realizar suas pesquisas. Ela também serve como modelo para o futuro da provisão de recursos computacionais de alto desempenho ou em grande escala para o setor produtivo.

Na América Latina, a realidade de infra-estrutura e de formação de fontes de informação científica está sendo marcada por duas iniciativas: a Rede CLARA e a Rede ScienTI, respectivamente.

Em termos de infra-estrutura de comunicação na região, a Rede CLARA constitui marco importante para uma maior integração entre as redes nacionais acadêmicas e de pesquisa da região. As comunidades de pesquisa latino-americanas e européias se beneficiarão desta nova infraestrutura de comunicação de dados, com novas possibilidades de troca rápida de informações. Além disso, ao facilitar o desenvolvimento e implantação de redes onde elas ainda não existiam e ao gerar 
oportunidades para a construção de uma agenda de pesquisa de interesse regional e global, CLARA abre novas perspectivas e possibilidades para seus usuários numa parceria que cumpre um importante papel na construção do futuro latino-americano em ciência e educação.

A Rede ScienTI, por sua vez, surge com a perspectiva de formação de conteúdos, fontes de informação e, especialmente, com a possibilidade de permitir conhecer os pesquisadores, grupos de pesquisa e a produção científica e tecnológica da América Latina. Em conseqüência, ScienTI deve promover um espaço de proveito direto à comunidade técnico-científica latino-americana. Até o momento, seu principal resultado está na formação das fontes nacionais, que já viabilizaram estudos inéditos em termos de indicadores de CT\&I na região.

CLARA e ScienTI podem, especialmente se articuladas, estabelecer conectividade e conteúdos necessários à promoção do conhecimento técnico-científico na região latino-americana. Com isso, apontam-se caminhos de desenvolvimento baseados no conhecimento, geração de riqueza, redução de fronteiras à competitividade, inovação tecnológica e distribuição de renda. $\mathrm{O}$ papel de redes acadêmicas nesse contexto já se evidencia em países em desenvolvimento. Iniciativas como as redes CLARA e ScienTI podem trazer respostas semelhantes à região latinoamericana.

\begin{abstract}
Knowledge-based development produces wealth and opens the frontiers of competitiveness, technological innovation and wealth distribution. In developed countries the process is intrinsically bound to the ability in producing innovation and the dynamics of network knowledge construction. Within this process the academic and research communities participate effectively in the dynamics of knowledge and innovation, an environment strongly based on Information and Communication Technology (ICTs). However, when compared to the developed countries' dynamics, the formation of such communities in Latin America is rare. In this context CLARA (Latin American Cooperation in Advanced Networks) and ScienTI (Information and Knowledge Network in Science, Technology and Innovation) networks are strategies for the establishment, dissemination and foment of scientific knowledge in Latin America. CLARA network integrates national academy networks in Latin America linking some 700 universities and research centers in the area. ScienTI network establishes and links scientific information sources in eleven countries within the area. The formation of a gigantic Latin American research community linking researchers, projects, studies and researches has an important role in the building of the world of science and education of the future. The formation and the functioning of the networks will be discussed from the point of view of community's knowledge building in Latin America.
\end{abstract}

Key-words: technology and information; scientific community; academic and research networks; latin america. 


\section{Referências}

BALANCIERI, Renato. Análise de redes de pesquisa em uma plataforma de gestão em ciência e tecnologia: uma aplicação à plataforma lattes. 2004. Dissertação (Mestrado em Engenharia de Produção) - Universidade Federal de Santa Catarina, Florianópolis, 2004.

CABRAL, Adilson. CLARA e ALICE: conectando o Brasil ao mundo em redes de alta velocidade. Disponível em: <http://www.comunicacao.pro.br/setepontos/20/claralice.htm>. Acesso em: 25 jun. 2005.

CASTELLS, Manuel. A sociedade em rede. In: A era da informação: economia, sociedade e cultura. São Paulo: Paz e Terra, 1999.

CLARA - COOPERAÇÃO LATINO AMERICANA DE REDES AVANÇADAS. Disponível em: <http://www.redclara.net>. Acesso em: 12 jun. 2005.

DRUCKER, P. F. Administrando para o Futuro: anos 90 e a virada do século. Tradução de Nivaldo Montigelli Jr. São Paulo: Pioneira, 1992.

FREITAS JUNIOR, Olival de Gusmão. Um modelo de sistema de gestão do conhecimento para grupos de pesquisa e desenvolvimento. 2003. Tese (Doutorado em Engenharia de Produção) - Programa de Pós-Graduação em Engenharia de Produção, Universidade Federal de Santa Catarina, Florianópolis.

GERAQUE, Eduardo. Ligação direta com a Europa. Disponível em: <www.rnp.br/redes/clara>. Acesso em: 25 jun. 2005 .

GONÇALVES, Alexandre Leopoldo; UREN, Victoria; KERN, Vinícius Medina; PACHECO, Roberto Carlos dos Santos. Mining Knowledge from Textual Databases: an Approach Using Ontology-Based Context Vectors. In: INTERNATIONAL CONFERENCE ON ARTIFICIAL INTELLIGENCE AND APPLICATIONS - IASTED 2005, Innsbruck, Austria. v. I, p. 66-71.

MACEDO, Tonia Marta Barbosa. Redes informais nas organizações: a co-gestão do conhecimento. Revista Ciência da Informação, v. 28, n. 1, 1999.

MELO, L. C. P; MACHADO, I. As redes nacionais de educação e pesquisa - NRENs - como vetores de internacionalização da pesquisa na América Latina. In: WORKSHOP ON SCIENCE AND TECHNOLOGY INDICATORS, 6., 2004, Buenos Aires. Anais... Buenos Aires, 2004.

PACHECO, Roberto Carlos dos Santos; KERN, Vinícius Medina. Arquitetura conceitual e resultados da integração de sistemas de informação e gestão da ciência e tecnologia. Datagramazero, Rio de Janeiro, v. 4, n. 2, p. 1-11, 2003.

RNP - REDE NACIONAL DE PESQUISA. Clara: Cooperação Latino-Americana de Redes Avançadas. Disponível em: <http://rnp.br/redes/clara>. Acesso em: 13 ago. 2005.

SCIENTI - REDE INTERNACIONAL DE FONTES DE INFORMAÇÃO E CONHECIMENTO EM CIÊNCIA, TECNOLOGIA E INOVAÇÃO. Disponível em: <http://www.scienti.net>. Acesso em: 10 ago. 2005.

SILVA, Edna Lúcia da. Rede científica e a construção do conhecimento. Revista Informação e Sociedade: Estudos. Disponível em: <http://www.informacaoesociedade.ufpb.br/1210205.html - 2k>. Acesso em: 26 jun. 2005.

SIMÕES, Nelson Simões. Entrevista concedida à Agência FAPESP. Disponível em: <http://www.rnp.br/redes/clara>. Acesso em: 26 jun. 2005

STANTON, Michael. Infra-estrutura para cooperação científica à distância. Disponível em: <http://www.rnp.br/noticias/imprensa/2005/not-imp-050327.html>. Acesso em 25 jun. 2005 a.

STANTON, Michael. As redes avançadas em 2004. Disponível em: <http://www.estadao.com.br/tecnologia/coluna/stanton/2003/dez/30/45.htm>. Acesso em: 25 jun. 2005 b.

TIDIA - TECNOLOGIA DA INFORMAÇÃO NO DESENVOLVIMENTO DA INTERNET AVANÇADA. Disponível em: <http://www.tidia.fm.usp.br/TIDIA.html>. Acesso em 12 jun. 2005. 
VELHO, Lea. Redes regionais de cooperação em C\&T e o Mercosul. Parcerias Estratrégicas, Brasília: (Ministério da Ciência e Tecnologia - Centro de Estudos Estratégicos), n.10, p.58-74, mar. 2001.

WEISZ, J.; ROCO, M. C. Redes de Pesquisa e Educação em Engenharia nas Américas. FINEP, 1996.

\section{Dados completos de todos os autores:}

\section{Nome completo: Rejane Sartori}

Filiação institucional: Programa de Pós-Graduação em Engenharia e Gestão do Conhecimento Universidade Federal de Santa Catarina (UFSC/EGC); Universidade Estadual de Maringá (UEM); CESUMAR; FAFIJAN

Departamento: Engenharia e Gestão do Conhecimento (EGC/UFSC)

Função ou cargo ocupado: Acadêmica de doutorado

Endereço completo para correspondência (bairro, cidade, estado, país e CEP): AV. Guaíra, 538 Zona 07 - 87.020.050 - Maringá - PR

Telefones para contato: 44-3224-1401 - 44-9972-8118

e-mail: rejane@egc.ufsc.br

\section{Nome completo: Roberto Carlos dos Santos Pacheco}

Filiação institucional: Universidade Federal de Santa Catarina (UFSC).

Departamento: Departamento de informática e estatística (INE/UFSC); Programa de Pós-Graduação em Engenharia e Gestão do Conhecimento (EGC/UFSC)

Função ou cargo ocupado: Docente

Endereço completo para correspondência (bairro, cidade, estado, país e CEP): Campus Universitário - Trindade - 88040-900, Florianópolis, SC

Telefones para contato: 48-8806-7105

e-mail: pacheco@stela.org.br

Recebido para publicação em: 30/06/06

Aceito para publicação em: 06/09/06 